\title{
THE MAXIMAL IDEALS IN QUATERNION ORDERS
}

\author{
JOHN A. RILEY
}

\begin{abstract}
Let $R$ be a Noetherian, integrally closed local domain, and $\Lambda$ an $R$-order in a generalized quaternion algebra over the quotient field of $R$. In this note, it is proved that: (a) $\Lambda$ has at most two maximal ideals; and (b) in case $\Lambda$ does have exactly two maximal ideals, the corresponding residue class rings are commutative fields.
\end{abstract}

The classical theory of orders in simple algebras, as presented for example in Deuring [1], has been renovated and extended by Auslander and Goldman in their papers of 1960 [2] and [3]. One of their basic results is that in a maximal order, whose center is a discrete rank one valuation ring, the radical is the unique maximal twosided ideal. A natural question to ask is whether the same is true for maximal orders over arbitrary regular local rings. In this generality, however, the answer is in the negative; Ramras has shown this, in a very recent paper [4], by giving an example of a maximal order over a regular local ring of dimension two whose radical is not the only maximal two-sided ideal. Interestingly enough, his example concerns maximal orders in a quaternion division algebra, essentially the simplest case for orders whose centers are of dimension larger than one. Again, in his example of such an ill behaved maximal order, he finds that it has exactly two maximal ideals, and that each of these is actually a maximal left and right ideal, so that his order modulo its radical is a direct sum of two fields. The purpose of this note is to point out that this is the worst possible situation which can occur for orders in quaternion algebras: I will prove the following theorem.

Theorem. Let $R$ denote a Noetherian integrally closed local domain with quotient field $K$, and let $\Sigma$ be a (generalized) quaternion algebra over $K$. If $\Lambda$ is an $R$-order in $\Sigma$, then either

(a) the radical of $\Lambda$ is its unique maximal two-sided ideal, or else

(b) $\Lambda$ contains exactly two maximal ideals $Q, Q^{-}$which are maximal as one-sided ideals, so that $\Lambda / Q$ and $\Lambda / Q^{-}$are (commutative) fields.

To begin with, let me remind the reader (see, for example, [5]) that in a generalized quaternion algebra $\Sigma$ over the field $K$ one has an

Received by the editors March 2, 1970.

AMS 1970 subject classifications. Primary 16A18, 16A66, 16A46; Secondary 16A28, 16A12.

Key words and phrases. Orders in quaternion algebras, orders in simple algebras, quaternion algebras, maximal ideals, radical of an order.

Copyright (c) 1971, American Mathematical Society 
"involutory anti-automorphism" $x \rightarrow x^{-}$, such that the trace, Sp $x$ $=x+x^{-}$, of $x$, and the norm, $\mathrm{N} x=x \cdot x^{-}$, of $x$ belong to $K$. Further, each element $x$ of $\Sigma$ satisfies the equation: $x^{2}-x \operatorname{Sp} x+\mathrm{N} x=0$. If $x$ is integral over $R$, then Sp $x$ and $\mathrm{N} x$ belong to $R$.

Now suppose that $\Lambda$ is an $R$-order in $\Sigma$ ( $R$ a Noetherian, integrally closed local domain). If $l$ is a left ideal in $\Lambda$, the conjugate, $l^{-}$, of $l$ is the right ideal $l^{-}=\left\{x^{-} ; x \in l\right\}$. An ideal $l$ is self-conjugate if $l^{-}=l$. I will show first that the proper self-conjugate ideals are contained in the radical of $\Lambda$. Thus, let $x \in l$ and consider the ideal in $R$ generated by Sp $x$ and $\mathrm{N} x$. If this is all of $R$, then $1=a \operatorname{Sp} x+b \mathrm{~N} x$ for some elements $a, b \in R$, and so $1=a\left(x+x^{-}\right)+b x x^{-}=a x+(a+b x) x^{-}$. Since $x, x^{-}$are in $l$, so is 1 , contradicting the fact that $l$ is a proper ideal. Hence the ideal generated by $\operatorname{Sp} x$ and $\mathrm{N} x$ is contained in the radical of $R$, and thereforein the radical $N$ of $\Lambda$. Since $x^{2}=x \operatorname{Sp} x-\mathrm{N} x$, this shows that $x^{2} \in N$. It follows that $l$ is a nil ideal modulo $N$, and hence that $l$ is also nilpotent modulo $N$. Now $N$ is the intersection of the finitely many maximal ideals in $\Lambda$, so that the fact that a power of $l$ is contained in $N$ implies that $l$ itself is contained in $N$. I have proved:

Proposition A. The proper self-conjugate ideals in $\Lambda$ are contained in the radical.

Next, if $Q$ is a maximal two-sided ideal in $\Lambda$, then so is $Q^{-}$( $\Lambda$ being self-conjugate) and $Q Q^{-}$is a proper self-conjugate ideal. By Proposition A, $Q Q^{-}$is contained in $N$ and hence in each maximal ideal. If $P$ is any maximal ideal, then, $Q Q^{-} \subset P \Rightarrow Q=P$ or $Q^{-}=P$. Thus $Q$ and $Q^{-}$are the only maximal ideals; the radical is maximal if and only if $Q=Q^{-}$.

For convenience, let us say that an order is of type I if its radical is maximal, and of type II if there are two distinct maximal ideals, $Q$ and its conjugate, $Q^{-}$. I show now that if $\Lambda$ is of type II, then $Q, Q^{-}$ are actually maximal left (and right) ideals, so that $\Lambda / Q$ and $\Lambda / Q^{-}$ are division rings. To do this, let $l$ be a maximal left ideal. Then $l$ contains either $Q$ or $Q^{-}$; say $l \supseteq Q$. Thus $l^{-} \supseteq Q^{-}$, and $l l^{-} \supseteq l Q^{-}$. However, $l l^{-}$is a self-conjugate two-sided ideal, and is, further, a proper ideal: for if not, then $l l^{-}=\Lambda$, so that $1=\sum l_{i} m_{i}^{-}$for certain elements $l_{i}, m_{i}$ in $l$. Then $1=\sum l_{i}\left(\operatorname{Sp} m_{i}-m_{i}\right)=\sum l_{i}$ Sp $m_{i}-\sum l_{i} m_{i} \in l$, a contradiction. Thus by Proposition $\mathrm{A}, l-\subseteq N$.

Therefore $l Q^{-} \subseteq N$, and $l Q^{-} \subseteq Q$. Hence either $Q^{-} \subseteq Q$, which is impossible since we have assumed that $\Lambda$ is of type II, or else $l \subseteq Q$. It follows that $l=Q$ and hence that $l$ is two-sided. The conjugate, $l$, is also two-sided. We have proved: 
Proposition B. If $\Lambda$ is of type II, the maximal ideals are all twosided, and there are just two of them, $Q$ and its conjugate, $Q^{-}$.

Since the maximal ideals $Q, Q^{-}$are two-sided, $\Lambda / Q$ and $\Lambda / Q^{-}$are division rings. To complete the proof of our theorem, it only remains to be shown that $\Lambda / Q$ and $\Lambda / Q^{-}$are commutative. This will follow automatically if it can be shown that all commutators, $x y-y x$, of elements of $\Lambda$ are contained in the radical. I now prove this:

Proposition C. If $\Lambda$ is of type II, then the commutator ideal is contained in the radical.

Proof. The conclusion will follow if it can be shown that $x y-y x \in N$ for all $x, y \in \Lambda$. First, choose $x$ to be a nonunit in $\Lambda$. Then, since the maximal left ideals $Q, Q^{-}$are two-sided, $x \in Q \cup Q^{-}$. But then for any $y, x y-y x \in Q \cup Q^{-}$, also, and so $x y-y x$ is a nonunit, also. Now $(x y-y x)^{2}+\mathrm{N}(x y-y x)=0$, since $\mathrm{Sp}(x y-y x)=0$, so that $(x y-y x)^{2}$ is in the radical of $R$, and hence in $N$. Since the maximal ideals of $\Lambda$ are both one and two-sided, they are completely prime, in the sense that a product cannot belong to one of them without a factor belonging to the same one. Thus $x y-y x$ belongs to each, and hence to $N$. Now I show that for any $x, x y-y x$ is an element of $N$. Thus, let $x, y$ be arbitrary elements of $\Lambda$. Since $Q, Q^{-}$are the two maximal ideals in $\Lambda, \Lambda=Q+Q^{-}$, and $x=q+r^{-}$, for some two elements $q, r \in Q$. Then $x y-y x=q y-y q+r^{-} y-y r$, and since $q, r$ are nonunits, $q y-y q$ and $r^{-} y-y r^{-}$belong to $N$ by what we have just proved. Thus $x y-y x$ belongs to $N$, and hence all commutators belong to the radical. The commutator ideal therefore is contained in $N$ and the proposition is proved.

On the basis of this proposition, then, $\Lambda / Q$ and $\Lambda / Q^{-}$are commutative fields and the theorem itself is proved.

\section{REFERENCES}

1. M. Deuring, Algebren, Springer, Berlin, 1935.

2. M. Auslander and O. Goldman, Maximal orders, Trans. Amer. Math. Soc. 97 (1960), 1-24. MR 22 \#8034.

3. - The Brauer group of a commutative ring, Trans. Amer. Math. Soc. 97 (1960), 367-409. MR 22 \#12130.

4. M. Ramras, Maximal orders over regular local rings of dimension two, Trans. Amer. Math. Soc. 142 (1969), 457-479.

5. N. Bourbaki, Livre II: Algèbre. Chapitre 8: Modules et anneaux semisimple, Actualités Sci. Indust., no. 1261, Hermann, Paris, 1958, \$11, no. 2. MR 20 \#4576.

State University of New York, College at Plattsburgh, Plattsburgh, NEW YORK 12901 